\title{
Endocrine Disruptors and Epigenetic Transgenerational Disease Etiology
}

\author{
MICHAEL K. SKINNER \\ Center for Reproductive Biology, School of Molecular Biosciences, Washington State University, Pullman, Washington 99164
}

\begin{abstract}
Exposure to an environmental factor (e.g. endocrine disruptor) during embryonic gonadal sex determination appears to be epigenetically reprogram the male germ-line and subsequently promote transgenerational adult-onset disease. Disease phenotypes resulting from this epigenetic phenomenon include testis abnormalities, prostate disease, kidney disease, tumor development, and immune abnormalities. The epigenetic mechanism is hypothesized to involve the induction of new imprinted-like DNA sequences in the germ-line to transgenerationally transmit disease phenotypes. This epigenetic transgenerational disease mechanism provides a unique perspective from which to view adult onset disease and ultimately offers new insights into novel diagnostic and therapeutic strategies. (Pediatr Res 61: 48R-50R, 2007)
\end{abstract}

$\mathrm{E}$ pigenetics refers to changes in gene function within the genome that do not directly involve changes to the DNA sequences that regulate the genome. The DNA sequence of the genome is the essential building block of the individual and all species. Therefore, the stability of the genome sequence is extensive and is not readily mutated, modified, or altered. Epigenetic regulation of the genome therefore generally involves factors such as chromatin structure, histone modifications, and DNA methylation. Many environmental factors and toxicants have not been shown to directly modify the genome sequence, however, these factors can alter epigenetic factors such as DNA methylation or chromatin structure, which in turn impacts the genome epigenetically. A consideration of environment-genome interactions requires that epigenetic regulation be considered as a component of the molecular basis upon which the environmental factors interact with the genome.

Environmental exposures have been found to promote several transgenerational disease states or phenotypes (1). Generally, an embryonic or early postnatal exposure is required for these transgenerational phenotypes to occur. An example includes the ability of an embryonic diethylstilbestrol (DES) exposure to promote F2 generation female and male reproductive tract defects (2). Another example is the ability of embryonic nutritional defects (i.e. caloric restriction) to promote an F2 generation diabetes phenotype (3). The reproduc-

Received December 11, 2006; accepted January 24, 2007.

Correspondence: Michael K. Skinner, Ph.D., Center for Reproductive Biology, School of Molecular Biosciences, Washington State University, Pullman, WA 99164-4231; e-mail: skinner@mail.wsu.edu

This research was supported in part by grants from the National Institutes of Health.

DOI: $10.1203 / p d r .0 b 013 e 3180457671$ ibility and frequency of these disease phenotypes suggests they are likely epigenetic rather than due to DNA sequence mutations. The potential that these exposure phenotypes are epigenetic transgenerational phenotypes remains to be directly demonstrated, but suggest such a phenomenon may exist.

A transgenerational phenomenon can be defined as the ability of an acquired physiologic phenotype or disease to be transmitted to subsequent generations through the germ-line, such that the subsequent generation is not directly exposed to the environmental factor or toxicant. For example, the exposure of a gestating mother exposes the F0 generation mother, the F1 generation embryo and the germ-line of the F2 generation. Since multiple generations were exposed, the phenotypes in the F0, F1, and F2 generations could be due to toxicology of the direct exposure and not necessarily transmitted through an alternate mechanism. Therefore, in the above case the F3 generation would be the first unequivocal transgenerational generation not exposed. This does not rule out that the F2 generation of the examples provided are not a transgenerational phenotype, but simply points out that a limitation exists to this conclusion due to the direct exposure of the germ-line in the F2 generation. A multigenerational exposure, such as that of an exposed gestating mother, can transmit a phenotype due to the toxicology of the direct exposure, but a transgenerational phenotype involves the transmission of a phenotype independent of the direct exposure.

Recently, the observation was made that a transient exposure of an F0 generation gestating rat at the time of embryonic sex determination to an endocrine disruptor may promote an adult onset disease of spermatogenic defects and male subfertility. Research has demonstrated that $90 \%$ of all male progeny for four generations (F1-F4) developed these disease states after the direct exposure of the F0 gestating rat (Fig. 1) (4). This transgenerational phenotype was only transmitted through the male germ-line (sperm) and was not passed through the female germ-line (oocyte). Before $120 \mathrm{~d}$ of age, the primary disease phenotype was a male testis and spermatogenic cell defect $(4,5)$. Animals that were allowed to age up to $1 \mathrm{y}$ had a transgenerational disease phenotype that included the development of numerous disease states. These aged animals had the following disease state frequencies; $17 \%$ tumor development, $50 \%$ prostate disease, $40 \%$ kidney disease, $30 \%$ immune abnormalities, and $30 \%$ severe infertility in males from F1 to F4 generations (6). Female animals were also found to develop transgenerational disease but did not transmit the phenotype to subsequent generations. 


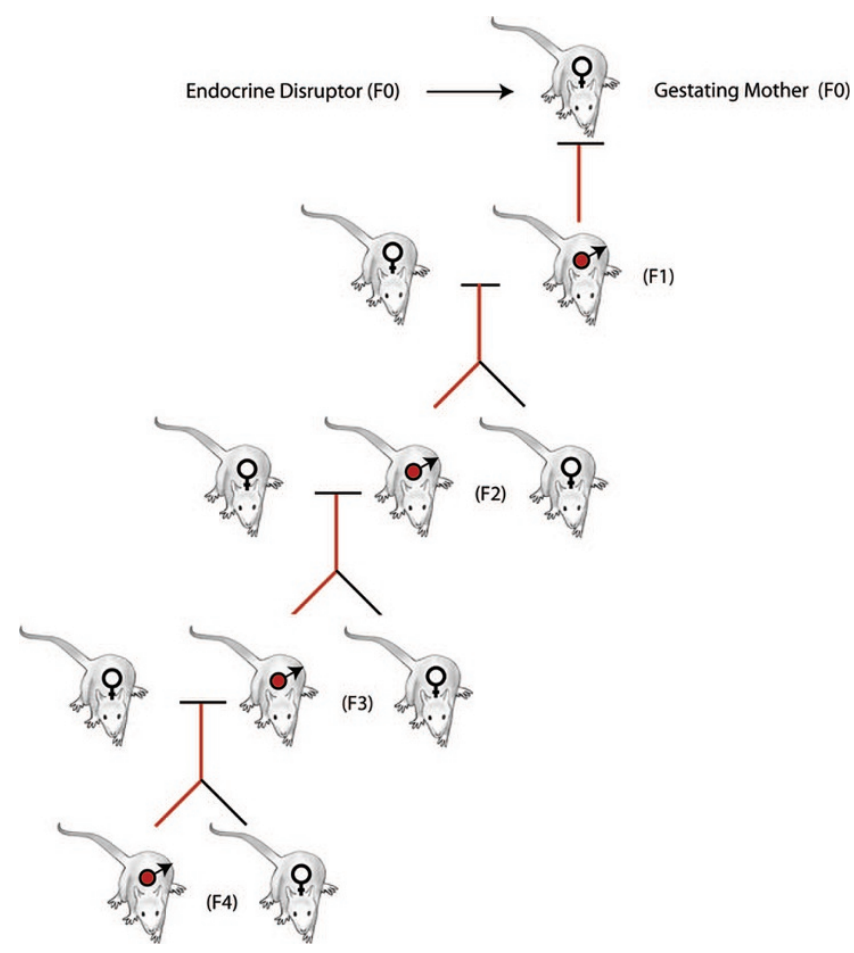

Figure 1. The postulated mechanism involved in endocrine disruptor actions during sex determination. Schematic of an F0 generation mother being transiently exposed to an endocrine disruptor, during embryonic sex determination and subsequent progeny through the male germ line, transgenerationally transmitting adult onset disease states.

The transgenerational (F1-F4) phenotype was induced by the endocrine disruptor vinclozolin. Vinclozolin is an antiandrogenic compound used as a fungicide in the fruit industry (e.g. wineries) (7). The phenotype can also be promoted by the pesticide methoxychlor, which is a mixture of estrogenic, antiestrogenic, and antiandrogenic metabolites (4). The ability of endocrine disruptors to promote adult onset disease has been previously reviewed (8). Endocrine disruptors are a large class of environmental toxicants ranging from plastics to pesticides (9). These environmental toxicants generally do not promote DNA sequence mutations that generally occur at a frequency $<0.01 \%$ (10). The frequency of the transgenerational phenotype described above (occurring in $30-90 \%$ of the sample population) also could not be attributed to DNA sequence mutations. Therefore, the hypothesis was developed that the transgenerational phenotype found is an epigenetic transgenerational phenotype resulting from changes in gene function that are not related to a DNA sequence mutation (Fig. 2) $(1,4)$.

The potential epigenetic mechanism hypothesized to be associated with this disease phenotype was investigated through analysis of the control and vinclozolin F3 generation sperm DNA. A methylation-sensitive restriction enzyme analysis identified DNA sequences/genes in the F3 vinclozolin generation sperm, each having an altered DNA methylation pattern. Several of these DNA sequences/genes were confirmed with bisulfite sequencing and shown to be transgenerational. Based on these findings, it has been suggested that the endocrine disruptor exposure during sex determination repro-

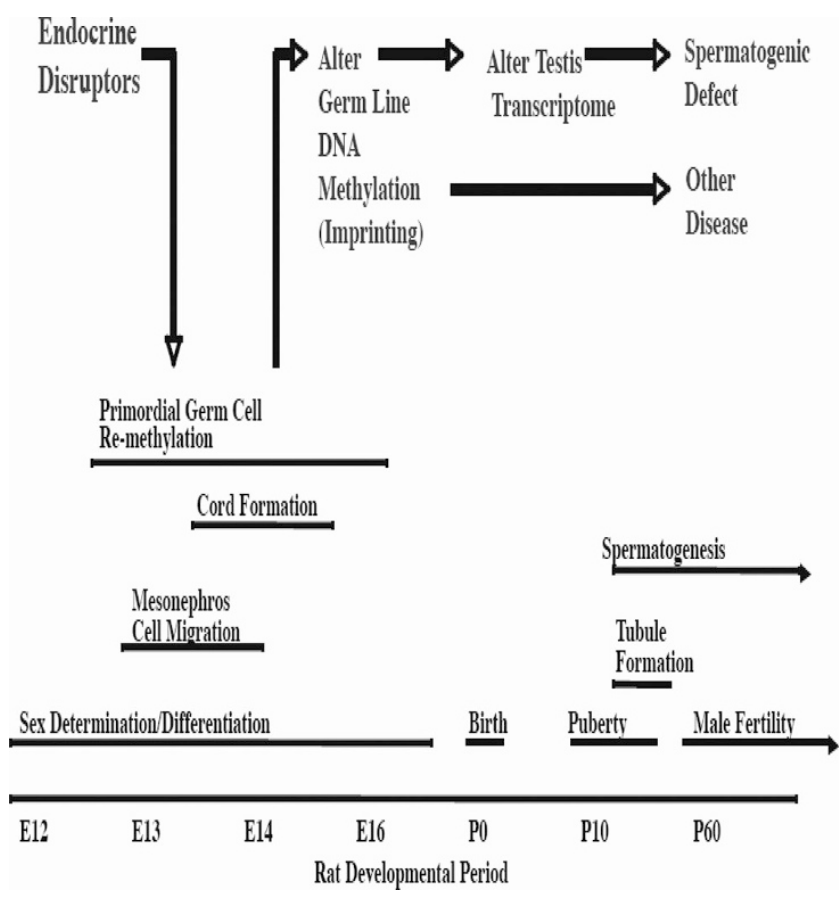

Figure 2. The postulated mechanism involved in endocrine disruptor actions during sex determination to promote alterations in imprinted-like DNA sequences/ genes in the male germ-line that then alter the transcriptions in developing organs to promote adult onset disease. $E$, embryonic day; $P$, postnatal day.

grammed the epigenetic coding/operations of the developing male germ-line and induced the presence of new imprintedlike genes/DNA sequences. These genes/DNA sequences were transmitted through the male germ-line (i.e. paternal allele) transgenerationally and promoted an alteration in the transcriptomes of different organs thereby inducing the disease phenotypes $(4,6)$. Sex determination has been shown to undergo a de-methylation and re-methylation process (11) such that a permanent reprogramming of DNA methylation of the germ-line may occur as a result of endocrine disruptor exposure (Fig. 1). The epigenetic transgenerational phenotype shown involves an endocrine disruptor (i.e. vinclozolin) exposure during embryonic sex determination resulting in an epigenetically reprogrammed (i.e. DNA methylation) male germ-line that induced new imprinted-like DNA sequences. These imprinted-like DNA sequences/genes may be transmitted through the male germ-line to all subsequent progeny (F1-F4) that then caused a dysregulation of the genome altering transcriptomes in a variety of organs and promoting transgenerational disease $(4,6)$.

This epigenetic transgenerational toxicology demonstrates a mechanism by which environmental toxicants may promote transgenerational phenotypes and adult-onset disease. A large number of studies have demonstrated that embryonic or postnatal exposures can induce adult onset disease $(8,9)$. The mechanism for this fetal basis of adult-onset disease, however, is largely unknown, but likely involves in part alterations in the genome epigenetically. Many adult-onset disease phenotypes are not transgenerational, but manifest in the individual exposed. These individual disease exposures and phenotypes may also involve epigenetic mechanisms. A recent study demonstrated a potential exposure to bisphenol A (BPA) 
promoted an alteration in DNA methylation of a number of genes and in the adult promoted a high frequency of prostate disease (12). Therefore, either an embryonic, postnatal, or adult exposure could cause an epigenetic event that then alters the physiology of a tissue and promotes disease. It is likely that rapidly developing organs that have the ability to alter a critical developmental step will be more sensitive to environmental exposures and epigenetic modifications. This epigenetic transgenerational mechanism is thought to be important for individual exposures and disease phenotypes. Therefore, a critical mechanism identified in the ability of an environmental exposure to induce an abnormal phenotype or physiology will likely involve epigenetic mechanisms in the toxicology of the agent or compound. Understanding toxicology on a molecular level is essential for the identification of biomarkers and the development of therapies to treat disease derived from environmental exposures. Epigenetics will be an important process to consider in the investigation of environmental exposures, environment-genome interactions, and the toxicology of specific compounds.

The epigenetic transgenerational disease phenotype described was due to an embryonic exposure promoting an adult-onset disease. The potential that an altered germ-line epigenome may be a causal mechanism for adult-onset disease must now be considered. The identification of novel epigenetic diagnostics and therapeutic strategies based on this unique disease etiology is anticipated to provide a significant advance in our understanding of disease phenotype transmission and development. The investigation of how an environmental compound (e.g. endocrine disruptor) can induce a transgenerational disease state may provide a mechanism to enhance our knowledge of how environmental factors influence disease independent of DNA sequence alterations. The elucidation of the role of epigenetics in environment-genome interactions will provide critical insights for environmental health and disease.

Acknowledgments. The authors thank Jill Griffin and Rochelle Pedersen for their assistance in the preparation of the manuscript.

\section{REFERENCES}

1. Anway MD, Skinner MK 2006 Epigenetic transgenerational actions of endocrine disruptors. Endocrinology 147:S43-S49

2. Newbold RR, Hanson RB, Jefferson WN, Bullock BC, Haseman J, McLachlan JA 1998 Increased tumors but uncompromised fertility in the female descendants of mice exposed developmentally to diethylstilbestrol. Carcinogenesis 19:1655-1663

3. Zambrano E, Martinez-Samayoa PM, Bautista CJ, Deas M, Guillen L, RodriguezGonzalez GL, Guzman C, Larrea F, Nathanielsz PW 2005 Sex differences in transgenerational alterations of growth and metabolism in progeny (F2) of female offspring $(\mathrm{F} 1)$ of rats fed a low protein diet during pregnancy and lactation. J Physiol 566:225-236

4. Anway MD, Cupp AS, Uzumcu M, Skinner MK 2005 Epigenetic transgenerational actions of endocrine disruptors and male fertility. Science 308:1466-1469

5. Anway MD, Memon MA, Uzumcu M, Skinner MK 2006 Transgenerational effect of the endocrine disruptor vinclozolin on male spermatogenesis. J Androl 27:868-879

6. Anway MD, Leathers C, Skinner MK 2006 Endocrine disruptor vinclozolin induced epigenetic transgenerational adult-onset disease. Endocrinology 147:5515-5523

7. Kelce WR, Monosson E, Gamcsik MP, Laws SC, Gray LE Jr 1994 Environmental hormone disruptors: evidence that vinclozolin developmental toxicity is mediated by antiandrogenic metabolites. Toxicol Appl Pharmacol 126:276-285

8. Gluckman PD, Hanson MA 2004 Developmental origins of disease paradigm: a mechanistic and evolutionary perspective. Pediatr Res 56:311-317

9. Heindel JJ 2005 The fetal basis of adult disease: role of environmental exposuresintroduction. Birth Defects Res A Clin Mol Teratol 73:131-132

10. Barber R, Plumb MA, Boulton E, Roux I, Dubrova YE 2002 Elevated mutation rates in the germ line of first- and second-generation offspring of irradiated male mice. Proc Natl Acad Sci U S A 99:6877-6882

11. Yamazaki Y, Mann MR, Lee SS, Marh J, McCarrey JR, Yanagimachi R, Bartolomei MS 2003 Reprogramming of primordial germ cells begins before migration into the genital ridge, making these cells inadequate donors for reproductive cloning. Proc Natl Acad Sci U S A 100:12207-12212

12. Ho SM, Tang WY, Belmonte de Frausto J, Prins GS 2006 Developmental exposure to estradiol and bisphenol A increases susceptibility to prostate carcinogenesis and epigenetically regulates phosphodiesterase type 4 variant 4 . Cancer Res 66:56245632 